\title{
Análise comparativa dos efeitos do diazepan, midazolam e propofol na contratilidade miocárdica: estudo em coração isolado de ratos
}

\author{
Carlos Geraldo Sobral de MEDEIROS*, José Carlos Dorsa Vieira PONTES*, \\ Otoni Moreira GOMES*
}

MEDEIROS, C. G. S.; PONTES, J. C. D.; GOMES, O. M. - Análise comparativa dos efeitos do diazepam, midazolam e propofol na contratilidade miocárdica: estudo em coraçōes isolados de ratos. Rev. Bras. Cir. Cardiovasc., 10 (4): 198-205, 1995.

RESUMO: A ação depressora dos benzodiazepínicos e do propofol relaciona-se às suas interferências diretas na fibra miocárdica. A presente investigação se propôs analisar a contratilidade miocárdica em coraçāo isolado de ratos tratados com diazepam, midazolam e propofol. Os parâmetros registrados foram: frequêencia cardíaca (bpm), tensāo miocárdica $(\mathrm{g})$ e fluxo coronariano $(\mathrm{ml} / \mathrm{min})$. Além das variaçōes da dT/ $\mathrm{dt}_{\text {max }}$, foram também analisadas aquelas ocorridas nos parâmetros acima citados, em grupos constituídos de dez coraçōes. Grupo I: perfundidos durante $\mathbf{3 0}$ minutos com soluçāo de Krebs-Hhenseleit (K-H); Grupo II: recebendo $50 \mathrm{mcg}$ de diazepam em $0,1 \mathrm{ml}$ de solução $\mathrm{K}-\mathrm{H}$, fazendo-se o registro dos parâmetros $1,3,5,10$, 15, 20, 25 e 30 minutos após; Grupo III diferiu do grupo anterior pela substituiçāo do diazepam por midazolam, na dose de $25 \mathrm{mcg} / 0,1 \mathrm{ml} \mathrm{K}-\mathrm{H}$; Grupo IV: propofol na dose de $25 \mathrm{mcg} / 0,1 \mathrm{ml} \mathrm{K}-\mathrm{H}$, efetuando-se os registros

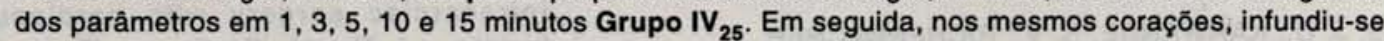
propofol na dose de $50 \mathrm{mcg} / 0,1 \mathrm{ml} \mathrm{K}-\mathrm{H}$, obtendo-se aqueles registros nos mesmos tempos acima (Grupo $\left.\mathrm{IV}_{\mathrm{50}}\right)$. A análise da contratilidade miocárdica ( $\mathrm{dT} / \mathrm{dt}_{\max }$ ) evidenciou decréscimos significativos nos grupos estudados exceto no Grupo I (Controle). As diminuiçōes observadas em g.seg ${ }^{-1}$ foram: Grupo II (diazepam), de $30,37 \pm 7,10$ para $21,50 \pm 6,03$ com redução de $29,21 \%$; Grupo III (midazolam), de $33,62 \pm 2,39$ para 24,62 $\pm 7,48$, redução de $26,77 \%$; Grupo IV (propofol) ${ }_{25}$, de $34,55 \pm 3,86$ para $28,50 \pm 5,97$, redução de $17,51 \%$ e Grupo IV (propofol) 50 , de $34,55 \pm 3,86$ para $27,57 \pm 5,86$, com redução de $20,20 \%$. Todas as drogas ensaiadas diminuíram a contratilidade miocárdica ( $\left.d T / d t_{\text {max }}\right) p<0,05$, sem diferença significante entre a intensidade com que cada uma, isoladamente, determinou tal efeito depressor $(p>0,05)$.

DESCRITORES: Diazepan na contratilidade miocárdica. Midazolam na contratilidade miocárdica. Propofol na contratilidade miocárdica. Miocárdio, contratilidade.

\section{INTRODUÇÃO}

Grande número de trabalhos já foi realizado com o objetivo de determinar a ação de drogas utilizadas em anestesia, na contratilidade miocárdica, com resultados por vezes controversos.
CHAI \& WANG ${ }^{4}$, em 1966, demonstraram que o diazepam atua no coração, deprimindo moderadamente a força contrátil, frequeência cardíaca e pressão arterial após infusão venosa em gatos anestesiados; concluíram que tais efeitos provinham

\footnotetext{
Trabalho realizado no Laboratório de Cirurgia do Serviço do Coração do Hospital São Francisco de Assis/CMC/SSVP. Belo Horizonte, MG, Brasil. Apresentado ao $22^{\circ}$ Congresso Nacional de Cirurgia Cardiaca. Brasília, DF, 30 de março a $1^{\circ}$ de abril, 1995.

- Do Hospital São Francisco de Assis, Belo Horizonte.

Endereço para correspondência: Carlos Geraldo S. de Medeiros. Rua XXIII, $n^{2} 115$, Q6, Bairro Nova Campo Grande. CEP: $79105-040$ - Campo Grande, MS, Brasil. Tel. (067) 763.1688
} 
MEDEIROS, C. G. S.; PONTES, J. C. D.; GOMES, O. M. - Análise comparativa dos efeitos do diazepam, midazolam e propofol na contratilidade miocárdica: estudo em coraçōes isolados de ratos.

Rev. Bras. Cir. Cardiovasc., 10 (4): 198-205, 1995.

de sua ação sobre os mecanismos centrais de controle cardiovascular.

PRINDLE et alii 17 , em 1970, não observaram tais ações depressoras relacionadas ao uso do diazepam, em preparações de músculos papilares de gatos. Por outro lado, ABEL et alii ${ }^{1}$, no mesmo ano, em modelo experimental canino, após infusão da droga, evidenciaram melhora da força contrátil do miocárdio e creditam tal efeito inotrópico positivo ao aumento do fluxo coronariano, fato posteriormente contestado por DANIEL ${ }^{7}$.

JONES et alii ${ }^{14}$, em 1979, publicaram rsultados de trabalhos experimentais em cães, onde demonstram efeito depressor do miocárdio após administração de midazolam.

RUIZ et alii ${ }^{20}$, em 1989 , observaram uma relação depressora dose/efeito do diazepam sobre o miocárdio e atribuíram tal relação à interferência negativa da droga no potencial de ação cálcio-dependente da fibra miocárdica, propondo que o diazepam possuía atividade antagonista do cálcio, residindo aí, provavelmente, seu mecanismo de atuação na função contrátil.

GONZALES et alii ${ }^{11}$, em 1990 , em coração isolado de ratos, observaram respostas diferentes da contratilidade miocárdica (aumento e diminuição), na dependência das concentrações de diazepam, e midazolam. MARTY \& NITENBERG ${ }^{15}$, nesse mesmo ano, contestaram a depressão miocárdica determinada pelo midazolam como causa do efeito hipotensor observado após seu uso, reacendendo antiga polêmica acerca da ação dos benzodiazepínicos na contratilidade miocárdica.

HERNÁNDES ${ }^{13}$, em 1991, e CARVALHO ${ }^{3}$, em 1993, mostraram, em corações isolados de ratos, inibição da contratilidade miocárdica após infusão de diazepam, reafirmando conceitos anteriormente exarados.

A afirmação de que o propofol determina hipotensão pela diminuição da contratilidade miocárdica 2, 18 foi contestada por outros autores, que atribuíram tal efeito à dose empregada, à velocidade de infusão, à diminuição da resistência vascular periférica, da freqüência cardíaca e/ou do fluxo coronariano $6,5,12,16$.

Os efeitos depressores acima citados, trazendo prejuízo ao desempenho do ventrículo esquerdo, podem aumentar a morbimortalidade de pacientes que se submetem a tratamento cirúrgico para correção de anomalias cardíacas.

O presente trabalho tem por objetivo avaliar os efeitos dos benzodiazepínicos (diazepam e midazolam) e do propofol na contratilidade miocárdica, em coração isolado de ratos.

\section{MATERIAL E MÉTODOS}

Foram estudados 40 ratos albinos, raça Wistar, provenientes do biotério do Serviço do Coração do Hospital São Francisco de Assis/CMC/SSVP, em Belo Horizonte/MG.

Após anestesia dos animais por inalação de éter sulfúrico em campânula fechada, procedeu-se à toracotomia ampla, heparinização com 500 unidades de heparina sódica em veia cava inferior, canulação da aorta com cateter plástico $18 \mathrm{~F}$, evitando-se lesão da valva aórtica e, finalmente, drenagem do ventrículo esquerdo pela introdução de cateter plástico $22 \mathrm{~F}$ em átrio esquerdo e valva mitral, exteriorizado pelo ápice do coração, de modo a não lesar vasos coronários importantes. A seguir, - coração foi excisado e conectado a sistema de Langendorff com líqüido de perfusão de KrebsHenseleit-K-H (Tabela 1), gaseificado com mistura de $95 \%$ de $\mathrm{O}_{2}$ e $5 \%$ de $\mathrm{CO}_{2}$, mantendo-se constantes a temperatura $(37,0 \pm 0,5$ graus Celcius $)$ mediante utilização de permutador térmico (FLVMEN Produtos Médicos Ltda) e a pressão de perfusão (90 cm de $\mathrm{H}_{2} \mathrm{O}$ ).

O coração, depois de perfundido por 15 minutos para recuperação e estabilização de suas atividades, foi conectado ao sistema de registro de contratilidade (Force Displacement Transducer, Model FT 03-GRASS Instrument Company), acoplado ao ápice do coração por dispositivo composto de roldanas com microrrolamentos.

Os registros da tensão miocárdica e freqüência cardiaca foram processados por biomonitor (BESE/ Bio-engenharia DH 073) e impressos em papel para análise e estudo comparativo, em impressora matricial EPSON LQ-1070. A medida do fluxo coronariano foi obtida pela aferição do volume minuto efluente das cavidades cardíacas, recolhido em frasco de vidro graduado.

Os 40 corações distribuídos em 4 grupos de 10 , assim discriminados: Grupo I (controle) - perfundido por 30 minutos, com registro dos parâmetros

TABELA 1 COMPOSIÇÃO DA SOLUÇÃO DE KREBS-HENSLEIT

\begin{tabular}{lr}
\hline Componentes & $\mathrm{mM} / \mathrm{L}$ \\
\hline $\mathrm{NaCl}$ & 126,0 \\
$\mathrm{NaHCO}_{3}$ & 25,0 \\
$\mathrm{KH}_{2} \mathrm{PO}_{4}$ & 1,2 \\
$\mathrm{KCl}$ & 4,8 \\
$\mathrm{MgSO}_{4}$ & 1,2 \\
$\mathrm{CaCl}_{2}$ & 2,5 \\
$\mathrm{Glicose}$ & 11,0 \\
\hline
\end{tabular}


MEDEIROS, C. G. S.; PONTES, J. C. D.; GOMES, O. M. - Análise comparativa dos efeitos do diazepam, midazolam e propofol na contratilidade miocárdica: estudo em coraçőes isolados de ratos. Rev. Bras. Cir. Cardiovasc., 10 (4): 198-205, 1995.

TABELA 2

VARIAÇÃO DA FREQÜÊNCIA CARDÍACA (bpm) - RESULTADOS EM MÉDIAS

\begin{tabular}{|c|c|c|c|c|c|c|c|c|c|c|c|c|c|c|c|c|c|c|}
\hline \multirow{2}{*}{$\frac{\text { Grupos }}{1}$} & \multicolumn{2}{|c|}{ Controle } & \multicolumn{2}{|c|}{1} & \multicolumn{2}{|c|}{3} & \multicolumn{2}{|c|}{5} & \multicolumn{4}{|c|}{$\begin{array}{c}\text { Perfodo } \\
\text { Min }\end{array}$} & \multicolumn{2}{|c|}{20} & \multicolumn{2}{|c|}{25} & \multicolumn{2}{|c|}{30} \\
\hline & 270 & $(29,22)$ & 270 & $(29,22)$ & 270 & $(29,22)$ & 260 & $\begin{array}{l}(21,79) \\
{[0,04]}\end{array}$ & 258 & $(23,63)$ & 247 & $\begin{array}{l}(23,34) \\
{[0,007]}\end{array}$ & 243 & $(20,28)$ & 240 & $(17,78)$ & 240 & $(17,78)$ \\
\hline$\|^{*}$ & 311 & $(25,44)$ & 304 & $(22,73)$ & 307 & $(19,85)$ & 307 & $(19,85)$ & 294 & $(30,34)$ & 289 & $(33,22)$ & 286 & $(29,83)$ & 280 & $(24,92)$ & 273 & $(38,27)$ \\
\hline III & 305 & $(41,00)$ & 311 & $(40,11)$ & 303 & $\begin{array}{l}(43,47) \\
{[0,04]}\end{array}$ & 294 & $\begin{array}{l}(43,56) \\
{[0,04]}\end{array}$ & 282 & $\begin{array}{l}(46,93) \\
{[0,009]}\end{array}$ & 279 & $(46,56)$ & 277 & $(47,44)$ & 267 & $\begin{array}{l}(46,61) \\
{[0,02]}\end{array}$ & 261 & $\begin{array}{l}(48,03) \\
{[0,04]}\end{array}$ \\
\hline $\mathrm{IV}_{25}$ & 317 & $(33,68)$ & 297 & $\begin{array}{l}(32,77) \\
{[0,008]}\end{array}$ & 286 & $\begin{array}{l}(29,84) \\
{[0,01]}\end{array}$ & 283 & $(29,66)$ & 264 & $\begin{array}{l}(21,57) \\
{[0,0008]}\end{array}$ & 260 & $(24,80)$ & - & & - & & - & \\
\hline $\mathrm{IV}_{50}$ & 317 & $(33,68)$ & 256 & $\begin{array}{l}(25,17) \\
{[0,0002]}\end{array}$ & 259 & $(14,94)$ & 255 & $(19,35)$ & 249 & $\begin{array}{l}(20,87) \\
{[0,04]}\end{array}$ & 237 & $\begin{array}{l}(18,21) \\
{[0,002]}\end{array}$ & - & & - & & - & \\
\hline
\end{tabular}

() Desvio Padrăo

[] Valores de $p<0,05$

- Năo houve variaçăo estatisticamente significativa nivel de significância 0,05

pesquisados após $1,3,5,10,15,20,25$ e 30 minutos; Grupo II (diazepam) - após os registros de controle, recebeu dose única de $50 \mathrm{mcg}$ de diazepam diluídos em $0,1 \mathrm{ml}$ de solução de $\mathrm{K}-\mathrm{H}$, injetada a montante da cânula inserida na aorta. Os registros foram feitos após $1,3,5,10,15,20,25$ e 30 minutos da infusão da droga; Grupo III (midazolam) - diferiu do grupo acima pela substituição do diazepam por midazolam, na dose única de $25 \mathrm{mcg}$ diluída em $0,1 \mathrm{ml}$ de solução de $\mathrm{K}-\mathrm{H}$; Grupo IV (propofol) - após os registros de controle, recebeu $25 \mathrm{mcg}$ de propofol diluídos em $0,1 \mathrm{ml}$ de solução de $\mathrm{K}-\mathrm{H}$, obtendo-se os parâmetros estudados após $1,3,5,10$ e 15 minutos da sua administração $\left(\mathrm{IV}_{25}\right)$; logo em seguida, $50 \mathrm{mcg}$ de propofol diluídos em $0,1 \mathrm{ml}$ de solução de $\mathrm{K}-\mathrm{H}$ foram infundidos, registrando-se os mesmos parâmetros em idênticos intervalos de tempo $\left(\mathrm{IV}_{50}\right)$.

Foram estudadas as variações ocorridas na freqüência cardíaca (bpm), tensão miocárdica (g), velocidade máxima de encurtamento da fibra miocárdica-dT/dt $t_{\max }\left(\mathrm{g} \mathrm{seg}^{-1}\right)$ e do fluxo coronariano $(\mathrm{ml} / \mathrm{min})$. Os resultados foram submetidos a análise de variância e teste $T$ de Student. O nível de significância foi de 0,05 .

\section{RESULTADOS}

Como demonstram os resultados em médias expostos na Tabela 2, a freqũência cardíaca diminuiu de forma significante nos grupos estudados, exceto no Grupo II (diazepan). Nos demais, observaram-se as seguintes variações, em batimentos por minuto (BPM): Grupo I (controle), de $270 \pm$ 29,22 para $240 \pm 17,78$ com redução percentual de $11,11 \%$, mais acentuadas nos minutos $5^{\circ}(p<0,04)$ e $15^{\circ}$ ( $p<0,007$ ); Grupo III (midazolam), de $305 \pm$ 41,00 para $261 \pm 48,03$, com redução de $14,43 \%$, ocorrendo os decréscimos mais significantes nos

TABELA 3

VARIAÇÃO DA TENSÃO MIOCÁRDICA (g) - RESULTADOS EM MÉDIAS

\begin{tabular}{|c|c|c|c|c|c|c|c|c|c|c|c|c|c|c|c|c|c|c|}
\hline \multirow{2}{*}{ Grupos } & \multicolumn{2}{|c|}{ Controle } & \multicolumn{2}{|c|}{1} & \multicolumn{2}{|c|}{3} & \multicolumn{2}{|c|}{5} & \multicolumn{4}{|c|}{$\begin{array}{c}\text { Periodo } \\
\text { Min }\end{array}$} & \multicolumn{4}{|c|}{$20 \quad 25$} & \multicolumn{2}{|c|}{30} \\
\hline & 3,20 & $(0,40)$ & 3,20 & $(0,40)$ & 3,20 & $(0,40)$ & 3,12 & $(0,28)$ & 3,15 & $(0,27)$ & 3,14 & $(0,27)$ & 3,12 & $(0,21)$ & 3,10 & $(0,19)$ & 3,13 & $(0,12)$ \\
\hline \multirow[t]{2}{*}{ II } & 2,43 & $(0,56)$ & 2,14 & $(0,63)$ & 2,17 & $(0,56)$ & 2,10 & $(0,62)$ & 2,01 & $(0,60)$ & 1,93 & $(0,49)$ & 1,85 & $(0,46)$ & 1,75 & $(0,47)$ & 1,72 & $(0,48)$ \\
\hline & & & & {$[0,003]$} & & & & & & & & {$[0,05]$} & & {$[0,03]$} & & {$[0,01]$} & & {$[0,04]$} \\
\hline III & 2,69 & $(0,19)$ & 2,66 & $(0,21)$ & 2,52 & $(0,27)$ & 2,43 & $(0,38)$ & 2,31 & $(0,48)$ & 2,19 & $(0,51)$ & 2,15 & $(0,54)$ & 2,05 & $(0,58)$ & 1,97 & $(0,60)$ \\
\hline \multirow[t]{2}{*}{$\mathrm{IV}_{25}$} & 2,80 & $(0,39)$ & 2,59 & $(0,38)$ & 2,51 & $(0,35)$ & 2,42 & $(0,35)$ & 2,35 & $(0,42)$ & 2,32 & $(0,43)$ & - & & - & & - & \\
\hline & & & & {$[0,005]$} & & {$[0,03]$} & & {$[0,01]$} & & & & & tha & thy & & int & & \\
\hline \multirow[t]{2}{*}{$\mathrm{IV}_{50}$} & 2,80 & $(0,39)$ & 2,33 & $(0,44)$ & 2,28 & $(0,46)$ & 2,34 & $(0,48)$ & 2,21 & $(0,49)$ & 2,24 & $(0,52)$ & $\cdot$ & & - & & - & \\
\hline & & & & {$[0,003]$} & & & & & & & & & & & & & & \\
\hline
\end{tabular}

( ) Desvio Padrăo

I] Valores de $p<0,05$

- Năo houve variaçăo estatisticamente significativa nivel de significância 0,05 
MEDEIROS, C. G. S.; PONTES, J. C. D.; GOMES, O. M. - Análise comparativa dos efeitos do diazepam, midazolam e propofol na contratilidade miocárdica: estudo em coraçōes isolados de ratos.

Rev. Bras. Cir. Cardiovasc., 10 (4): 198-205, 1995.

$26,77 \%$ e variaçōes significantes nos minutos $3^{2}$ $(p<0,006), 5^{\circ}(p<0,04), 10^{\circ}(p<0,01), 15^{\circ}(P<0,001)$, $20^{\circ}(\mathrm{p}<0,01), 25^{\circ}(\mathrm{p}<0,03)$ e $30^{\circ}(\mathrm{p}<0,03)$; Grupo IV com propofol na dose de $25 \mathrm{mcg}\left(\mathrm{IV}_{25}\right)$, de 34,55 $\pm 3,86$ para $28,50 \pm 5,97$ com redução de $17,51 \%$; as diminuições significativas $\mathrm{da} \mathrm{dT} / \mathrm{dt}_{\max }$ ocorreram nos minutos $1^{\circ}(p<0,0001), 3^{\circ}(p<0,03)$ e $5^{\circ}(p<0,01)$ mantendo-se neste platô mais baixo sem variações significativas, nos minutos $10^{\circ}$ e $15^{\circ}$. Grupo IV com propofol na dose de $50 \mathrm{mcg}\left(\mathrm{IV}_{50}\right)$, de $34,55 \pm 3,86$ para $27,57 \pm 56,86$ com redução de $20,20 \%$, observando-se diminuição significativa do parâmetro em apreço, nos minutos $1^{\circ}(p<0,0008)$ e $5^{\circ}(p<0,02)$.

O fluxo coronariano, conforme resultados em médias, especificados na Tabela 5 , e expresso em $\mathrm{ml} / \mathrm{min}$, apresentou diminuições estatisticamente significativas em todos os grupos, como se segue: Grupo I (controle), de $13,30 \pm 2,86$ para $11,50 \pm$ $2,68 \mathrm{com}$ redução de $15,53 \%$ e variações significativas nos minutos $15^{\circ}(p<0,007)$ e $25^{\circ}(p<0,02)$; Grupo II (diazepam), de $8,85 \pm 2,46$ para $6,55 \pm$ 2,22 com redução de $25,99 \%$; as variações são significativas nos minutos $1^{\circ}(p<0,04), 3^{\circ}(p<0,001)$, $10^{\circ}(p<0,0004), 15^{\circ}(p<0,01)$ e $25^{\circ}$ ( $\left.p<0,01\right)$; Grupo III (midazolam), de $7,20 \pm 0,63$ para $4,80 \pm 0,67$ com redução de $33,33 \%$, sendo os decréscimos significantes durante o experimento, exceto no $30^{\circ}$ minuto ( $p<0,29)$; Grupo IV com propofol na dose de $25 \mathrm{mcg}\left(\mathrm{IV}_{25}\right)$ e com propofol na dose de 50 mcg $\left(\mathrm{IV}_{50}\right)$, exceto no $3^{2}$ minuto deste último, todos os demais tempos evidenciaram decréscimos significantes do parâmetro em pauta, sendo uma redução de $43,59 \%$ para o $I_{25}$ e de $47,69 \%$ para - IV $_{50}$.

O Gráfico 1 ilustra as alterações apresentadas pela contratilidade miocárdica (dT/dt), ao longo de todo o experimento entre os grupos estudados, em valores percentuais.

\section{COMENTÁRIOS}

A injeção de 7-cloro-1,3-diidro-1-metil-5-fenil-2H1,4-benzodiazepin-2-ona (diazepam), na dose de 50 microgramas, determinou reduções significantes $(p<0,05)$ da tensão miocárdica, $d T / d t_{\max }$ e fluxo

\section{GRÁFICO 1}

VARIAÇÃO PERCENTUAL DA CONTRATILIDADE MIOCÁRDICA (dT/dt) MUDANÇAS NA CONTRATILIDADE MIOCÁRDICA (dT/dt), EM VALORES PERCENTUAIS, NOS DIVERSOS GRUPOS ESTUDADOS

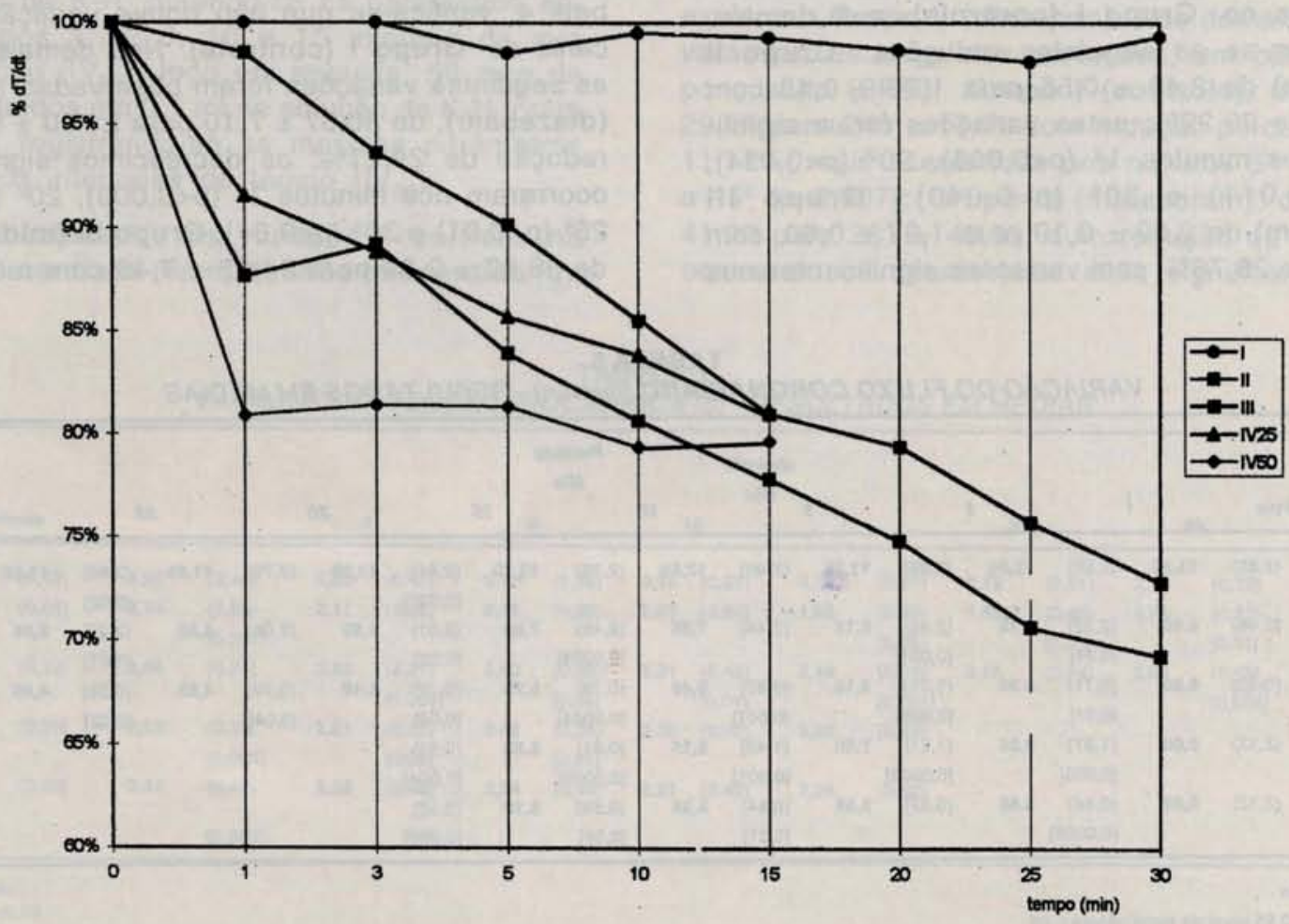


MEDEIROS, C. G. S.; PONTES, J. C. D.; GOMES, O. M. - Análise comparativa dos efeitos do diazepam, midazolam e propofol na contratilidade miocárdica: estudo em coraçōes isolados de ratos.

Rev. Bras. Cir. Cardiovasc., 10 (4): 198-205, 1995.

coronariano, quando cotejadas com os valores inicias (controles), com queda para os seguintes valores percentuais, respectivamente: $70,84 \%, 69,21 \%$ e $73,50 \%$. É interessante assinalar que estas modificações do desempenho cardíaco ocorreram com dose relativamente baixa da droga, que, relacionada ao fluxo coronariano no momento da infusão $(8,85 \mathrm{ml} / \mathrm{min})$, correspondeu a $5,64 \mathrm{mcg} / \mathrm{ml}$ estando, portanto, dentro da faixa de concentrações de uso clínico corrente compreendida entre 5 e 32 $\mathrm{mcg} / \mathrm{ml}^{8}$. Os resultados por nós obtidos com o uso do diazepam confirmam as conclusōes de CHAI \& WANG ${ }^{4}$, em 1966, TAYLOR et alii ${ }^{22}$, em 1970 , DANIEL et alii ${ }^{7}$, em 1975, SUGIMOTO et alii ${ }^{21}$, em 1978, GONZALES et alii ${ }^{11}$, em 1990 , e CARVALHO 3, em 1993. Diferem, contudo, das conclusões de PRINDLE et alii ${ }^{17}$ e ABEL et alii ${ }^{1}$, ambos em 1970 , que demonstraram ação inócua ou até mesmo inotrópica positiva após uso do diazepam.

A infusão de [8-cloro-6 (2-fluorofenol)-1-metil$4 \mathrm{H}$-imidazol $(1,5-\mathrm{a})(1,4)]$ benzodiazepina (midazolam), na dose de $25 \mathrm{mcg}$, determinou alterações significativas $(p<0,05)$ da freqüência cardíaca, tensão miocárdica, $\mathrm{dT} / \mathrm{dt}_{\max }$ e fluxo coronariano, com declínio para os seguintes valores percentuais relacionados aos controles, respectivamente: $85,33 \%, 72,75 \%, 72,75 \%$ e $66,69 \%$. Levando-se em conta o fluxo coronariano médio de $7,20 \mathrm{ml} / \mathrm{min}$, no momento da injeção da droga, a dose empregada correspondeu a $3,47 \mathrm{mcg} / \mathrm{ml}$, aquém, portanto, da concentração plásmática determinada por dose de $0,3 \mathrm{mg} \mathrm{kg}^{-1}$, de uso corrente na prática anestésica

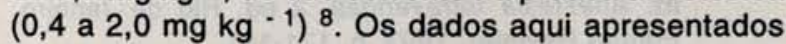
confirmam os resultados obtidos por JONES et alii ${ }^{14}$, em 1979, e por REVES ef alii ${ }^{19}$, em 1984, que descreveram efeitos depressores do miocárdio decorrentes do uso de midazolam. Não repetimos, por outro lado, os resultados publicados por MARTY et alii ${ }^{15}$, em 1990, que não observaram aquela ação depressora que justificasse o efeito hipotensor determinado pelo uso do midazolam.

A injeção de 2,6-diisopropilfenol (propofol) na dose de 25 microgramas, considerado o fluxo coronariano médio naquele momento $(9,75 \mathrm{ml} / \mathrm{min})$, resultou em alterações significativas $(p<0,05) \mathrm{da}$ freqüência cardíaca, tensão miocárdica, $d T / d t_{\max } e$ fluxo coronariano, com declínio para os seguintes valores percentuais, cotejados com os valores de controle, respectivamente: $82,54 \%, 82,91 \%, 82,12 \%$ e $58,37 \%$. A infusão de propofol na dose de 50 microgramas, quando correlacionado com os registros controles, diminuiu ainda mais os valores supracitados. Após esta segunda dose, os novos valores percentuais atingidos, referentes aos parâmetros acima mencionados foram, respectivamente: $75,76 \%, 79,52 \%, 79,60 \%$ e $55,01 \%$. As modificações assinaladas ocorreram com doses (25 $\mathrm{mcg}$ e $50 \mathrm{mcg}$ ) que relacionadas aos fluxos coronarianos já referidos, no momento de suas injeções, determinaram concentrações de $2,57 \mathrm{mcg} /$ $\mathrm{ml}$ e $9,09 \mathrm{mcg} / \mathrm{ml}$, respectivamente, bem abaixo, portanto, daquelas obtidas $(24$ a $30 \mathrm{mcg} / \mathrm{ml}) \mathrm{com}$ o uso de doses usualmente empregadas em anestesia (1,6 a $2,5 \mathrm{mg} \mathrm{kg-1})^{9}$. Estes resultados confirmam aqueles publicados por BRUSSEL et alii ${ }^{2}$, em 1989 , PUTTICK et alii ${ }^{18}$, em 1992, que relataram efeitos depressores do miocárdio pelo uso do propofol. Por outro lado, diferem dos resultados apresentados por COATES et alii ${ }^{6}$, em 1985, CLAEYS et alii ${ }^{5}$, em 1988, GOODCHILD et alii ${ }^{12}$, em 1989 e MOUREN et alii ${ }^{16}$, em 1994, que não evidenciaram alterações significantes da contratilidade miocárdica, ou não observaram depressão miocárdica que justificasse os efeitos hipotensores resultantes do uso da droga.

Possivelmente em doses mais baixas, o uso clínico destas drogas não manifeste a nocividade de seus efeitos sobre a contratilidade miocárdica, face aos mecanismos fisiológicos de homeostasia decorrentes da interação entre o desempenho cardíaco e o sistema neuro-humoral de controle circulatório, notadamente a atividade simpática e a liberação adrenérgica. Este efeito depressor aqui observado, no entanto, pode tornar-se muito mais evidente em situações clínicas e experimentais que associem o emprego de outras substâncias cardioinibidoras, como as soluções cardioplégicas, justificando, neste caso, a "síndrome do coração bêbado" descrita por GOMES et alli ${ }^{10}$, a partir de investigaçōes experimentais. Mais importante, é a diferente sensibilidade dos corações gravemente comprometidos, especialmente operados, obviamente mais susceptíveis à depressão por essas drogas.

Pode-se concluir, da presente investigação, que todas as drogas ensaiadas (diazepam, midazolam e propofol) deprimiram de forma significante $(p<0,05)$ a função contrátil do miocárdio. A análise estatística não mostrou diferenças significativas $(p>0,05)$ entre a intensidade com que cada droga determinou sua ação depressora no coração isolado. A diminuição da contratilidade miocárdica determinada pelo propofol na dose de 50 microgramas foi maior que a produzida pela mesma droga na dose de 25 microgramas, com variações estatisticamente significativas $(p<0,05)$. 
MEDEIROS, C. G. S.; PONTES, J. C. D.; GOMES, O. M. - Análise comparativa dos efeitos do diazepam, midazolam e propofol na contratilidade miocárdica: estudo em coraçōes isolados de ratos.

Rev. Bras. Cir. Cardiovasc., 10 (4): 198-205, 1995.

\section{RBCCV 44205-278}

MEDEIROS, C. G. S.; PONTES, J. C. D. V.; GOMES, O. M. - Comparative analysis of diazepam, midazolam and propofol effects in myocardial contractility: isolated rat's hearts study. Rev. Bras. Cir. Cardiovasc., 10 (4): 198-205, 1995.

ABSTRACT: Cardiac rate, myocardial tension, coronary flow and myocardial contractjlity (dT/dt) variantions were registered in a population of fourty Wistar rats. In all animals, after ether anesthesia, the hearts were removed and perfused with $95 \% \mathrm{O}_{2}$ and $5 \% \mathrm{CO}_{2}$ enriched Krebs-Henseleit $(\mathrm{K}-\mathrm{H})$ solution, under $90 \mathrm{~cm}_{2} \mathrm{O}$ constant pressure and temperature of $37,0 \pm 0.5^{\circ} \mathrm{C}$, until complete stabilization, for control values registration. The hearts were distributed into four groups: Group I were perfused with $\mathrm{K}-\mathrm{H}$ solution, gathering the data of cardiac performance after $1,3,5,10,15,20,25$, and 30 minutes. In the other three groups, these data were also registered in the same times, after they were treated with $50 \mathrm{mcg}$ of diazepam (Group II), $25 \mathrm{mcg}$ of midazolam (Group III) and $25 \mathrm{mcg}$ followed by $50 \mathrm{mcg}$ of propofol (Group IV 25 and IV $\mathrm{V}_{50}$ ). The cardiac rate underwent significant variation in the groups estudied except Group II. Myocardial tension varied to a negative degree in the groups, except for Groups I and IV (propofol $50 \mathrm{mcg}$ ). In relation to myocardial contractility (dT/ dt), except Group I, all groups underwent decreases. The coronary flow exhibited decreases in all groups studied. The observed variations were statistically significant $(p<0.05)$. It is concluded that the drugs tested (diazepam, midazolama and propofol) determine statistically significant decrease of the myocardial contractility, without difference concerning the intensity of depression degree among all studied groups.

DESCRIPTORS: Diazepan in myocardial contractility. Midazolam in myocardial contractility. Propofol in myocardial contractility. Myocardial contractility.

\section{REFERÊNCIAS BIBLIOGRÁFICAS}

1 ABEL, R. M.; REIS, R. L.; STAROSCIK, R. N. - The pharmacological basis of coronary and sistemic vasodilator actions of diazepam. Br. J. Pharmacol., 39: $261-274,1970$.

2 BRÜSSEL, R. M.; THEISSEN, J. L.; VIGFUSSON, G.; LUNKENHEIMER, P. P.; VAN AKEN, H.; LAWIN, P. - Hemodynamic and cardiodynamic effects of propofol and etomidate: negative inotropic properties of propofol. Anesth. Analg., 69: 35-40, 1989.

3 CARVALHO, J. I. - Efeito do 7-cloro-1, 3-dihidro-1-metil5-fenil-2h-1, 4-benzodiazepin-2-ona (diazepam) na contratilidade miocárdica e na resposta à dopamina: estudo em corações isolados de ratos. Belo Horizonte, 1993. Tese Mestrado - Curso de Pós-Graduação em Cardiologia e Cirurgia Cardiovascular do Serviço do Coração do Hospital São Francisco de Assis/CMC/SSVP].

CHAI, C. Y. \& WANG, S. C. - Cardiovascular actions of diazepam in the cat. J. Pharmacol. Exp. Ther. 154: 271-280, 1966 .

5 CLAEYS, M. A.; GEPTS, E.; CAMU, F. - Haemodynamics changes during anaesthesia induced and maintained with propofol. Br. J. Anaesth, 60: 3-9, 1988.

COATES, D. P.; PRYS-ROBERTS, C.; SPELINA, K. R.; MONK, C. R.; NORLEY, I. Propofol (diprivam) by intravenous infusion with nitrous oxide: doses requirements and haemodynamics effects. Postgraduate Med. J., 61 (Supl. 3): 76-79, 1985.

7 DANIEL, H. B. - Cardiovascular effects of diazepam and chlorodiazepoxide. Europ. J. Pharmacol., 32: 5865, 1975.

8

DUNDEE, J. W. \& WYANT, G. M. - As Benzodiazepinas. In: Anestesia Intravenosa. 2 ed. Rio de Janeiro, Editora Revinter, 1993. p. 171-191.

DUNDEE, J. W. \& WYANT, G. M. - Propofol. In: Anestesia intravenosa, 2. ed. Rio de Janeiro, Editora Revinter, 1993. p. 159-170.

GOMES, O. M. \& MORAES, D. J. - Síndromes de proteção miocárdica. Coração, 2: 3-5, 1992.

GONZALES, R.; ZEEGER, A.; EIGENHUIS, J. J.; LEEWIN, R. S.; VAN WILGENBURG, H. A. Comparative study of diazepam and midazolam induced actions in isolated rat hearts. Europ. J. Pharmacol., 183: 2082, 1190.

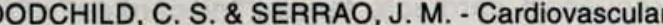
effects of propofol in the anaesthetized dog. Br.J. Anaesth., 63: 87-92, 1989.

HERNÁNDEZ J. - The negative inotropic effect of diazepam in rat right ventricular strips. J. Pharm. Pharmacol., 43: 879-882, 1991.

ONES, D. J.; STEHLING, L. C.; ZANDER, H. L. Cardiovascular responses to diazepam and midazolam maleate in the dog. Anesthesiology, 51: 430-434, 1979. 
MEDEIROS, C. G. S.; PONTES, J. C. D.; GOMES, O. M. - Análise comparativa dos efeitos do diazepam, midazolam e propofol na contratilidade miocárdica: estudo em coraçōes isolados de ratos. Rev. Bras. Cir. Cardiovasc., 10 (4): 198-205, 1995.

and flumazenil in cardiovascular diagnostic and therapeutic procedures. Acta Anaesthesiol. Scand., 92 (Supl.): 33-34, 1990.

MOUREN, S.; BARON, J. F.; ALBO, E.; SZEKELY, B.; ARTHAUD, M.; VIARS, P. - Effects of propofol and thiopental on coronary blood flow and myocardial performance in an isolated rabbit heart. Anesthesiology, 80: 34-41, 1994.

17 PRINDLE, K. H.; GOLD, H. K.; CARDON, P. V.; EPSTEIN, S. E. - Effects of psycopharmacologic agents on myocardial contractility. J. Pharmacol. Exp. Ther., 173: 133-137, 1970.

18 PUTTICK, A. M.; DIEDERICKS, J.; SEAR, J. W.; GLEN J. B.; FOEEX, P.; RYDER, W. A. - Effects of graded infusion rates of propofol on regional and global left ventricular function in dog. Br. J. Anaesth., 69: 375$381,1192$.
19 REVES, J. G.; KISSIN, I.; FOURNIER, S. - Negative inotropic effects of midazolam. Anesthesiology, 60: 517-518, 1984.

20 RUIZ, F.; HERNÁNDEZ, J.; PÉREZ, D. - The effect of diazepam on ventricular automaticity induced by local injury: evidence of involviment of "peripheral-type" benzodiazepine receptors. J. Pharm. Parmacol., 41: 306-310, 1989.

21 SUGIMOTO, J.; NAGATA, M.; IKEDA, Y. - The effects of diazepam on rat isolated heart muscle. Clin. Exper. Pharmacol. Physiol., 5: 655-663, 1978.

22 TAYLOR, P.L.; DANNIEL, H.B.; BAGWELL, E.E. Studies on the myocardial and metabolic effects of diazepam in the dog. Pharmacologist, 12: 23332335, 1970. 